\title{
Intra-neural ceroid-like pigment following the treatment of lepromatous leprosy with clofazimine (B663; Lamprene)
}

\author{
A C M C D O UGALL AND R L JONES \\ From the Department of Dermatology, Slade Hospital, Headington, Oxford
}

SUMMARY A 33 year old male Nigerian presented with widespread involvement of peripheral nerves, several of which were tender and painful. Nerve biopsies confirmed the presence of Mycobacterium leprae in both endoneurial and perineurial areas, mainly in foamy macrophages (Virchow cells), but there were also large accumulations of an amorphous, acid-fast and alcoholfast material which was not obviously of bacterial origin. Appropriate stains indicated that this had many characteristics of lipofuscin. Although not previously known, it was at this stage discovered that the patient had received treatment with anti-leprosy drugs nearly three yars before presentation in this country. One of these was clofazimine, an aniline aposafranine derivative known to produce a ceroid-like pigment in the tissues of patients treated with this drug for lepromatous leprosy.

As part of a programme directed towards the discovery of new drugs for the treatment of tuberculosis, clofazimine (B663: Lamprene) was first synthesised by Barry and his colleagues in Dublin in 1957. ${ }^{1}$ It did not in fact develop as a useful drug in the treatment of tuberculosis in humans but was soon shown to be of great value in leprosy, not only for the treatment of the bacterial infection but also for the suppression of immune complex reactions in the lepromatous form of this disease. Hastings et $a l^{2}$ have reviewed most of the important publications on its value in clinical leprosy, and more recently, (1978) Yawalkar and Vischer ${ }^{3}$ have published a monograph on clofazimine, covering its chemical composition and development, dosage, clinical use, complications and toxicity. It is now recommended by the World Health Organisation ${ }^{4}$ as one of the drugs for the combination with dapsone in the initial treatment of all new lepromatous patients and for combination with other drugs in the treatment of patients who are shown to be dapsone resistant.

Clofazimine is an aniline aposafranine which may produce red or reddish brown pigmentation not only in the lesions of leprosy, but also in the

Address for reprint requests: Dr AC McDougall, The Slade Hospital, Headington, Oxford OX3 $7 \mathrm{JH}$.

Accepted 25 September 1980 skin generally-a very obvious disadvantage for light-skinned patients. It has also been shown to accumulate as crystals in the lamina propria of the small bowel, the lymph nodes and in various other internal organs. Jopling ${ }^{5}$ has reviewed the literature of those exceptional cases in which such crystal accumulations appear to have been associated with abdominal pain, occasionally leading to laparotomy. Quite apart from crystal accumulation in tissues, it has however been known for many years that pigment can be seen in the tissues of patients taking this drug, even after the use of alcohol and other organic solvents in fixation and processing. Sakurai and Skinsnes ${ }^{6}$ have analysed this phenomenon, concluding that the residual pigment was closely related to ceroid.

We report the case of a patient under treatment in this country for lepromatous leprosy with rifampicin and dapsone, whose nerves contained not only Mycobacterium leprae, but also large accumulations of acid-fast, sudanophilic material in foam (Virchow) cells of both endoneurial and perineurial areas.

\section{Patient and methods}

A 33 year old male Nigerian was referred from Dublin to Leeds in June 1979, complaining of thickening of the palms and soles, together with pain and 116 
burning sensations in the limbs of approximately three years' duration. On examination several peripheral nerves were palpably enlarged and there were distal areas of anaesthesia to light touch on all four limbs. A clinical diagnosis of leprosy was made, and this was confirmed by finding acid-fast bacilli in slit skin smears and nasal mucus. Biopsy of a hyperpigmented area of skin from the back of the right thigh was however negative for infiltrate or bacilli, and a lymph node biopsy was also negative for bacilli, though it showed a large number of foamy histiocytes in the medulla. Routine examination and laboratory investigation was otherwise normal, apart from the radiological finding of cystic changes in the proximal phalanx of the fourth toe and the fifth metatarsal in the left foot. The patient completely denied a previous diagnosis of leprosy or any known treatment for this infection. He was started on rifampicin $600 \mathrm{mg}$ daily and dapsone $5 \mathrm{mg}$ daily and appears to have continued these drugs until arrival in Oxford in November, 1979, having left the Leeds area in the interval and been without clinical supervision for nearly two months. At that time he showed bilateral, almost symmetrical involvement of the ulnar, terminal, radial, median, lateral popliteal, posterior tibial and superficial peroneal nerves, with considerable enlargement, tenderness and pain. There was marked wasting of the thenar eminences, and of the first and second dorsal interossei on the right; dorsiflexion of the feet was bilaterally weak. There were areas of anaesthesia to light touch on all four limbs of an approximately glove and stocking type, the upper level being $3-4 \mathrm{~cm}$ below the elbows and knees. Vibration sense, deep pain, position sense and reflexes were normal. The skin showed absolutely no evidence of pigmentary change, infiltration or nodulation. Eyebrows, eyes, ears, nose, mouth, teeth, voice, breasts, and testicles were clinically normal. The skin of the palms and soles was bilaterally, symmetrically thickened and there was a suggestion of contracture of Dupuytren type over flexor tendons on the right side. A diagnosis of lepromatous leprosy in reaction was made and his nerve pain and tenderness brought under control by the addition of prednisolone $30 \mathrm{mg}$ daily while reducing rifampicin to $300 \mathrm{mg}$ daily and increasing dapsone to $25 \mathrm{mg}$ daily. A week later, on the strong presumption that rifampicin might be interfering with the full action of steroids, 78 rifampicin was stopped altogether, with progressive improvement in the patient's neurological state. In view of some difficulty with the full interpretation of a biopsy of auricular nerve taken on the patient's first presentation in this country, a repeat biopsy was now performed, from the right superficial peroneal nerve, above the ankle joint. This was fixed in buffered $10 \%$ formalin, embedded in wax and cut at 5 microns. $H$ and $E$ sections showed an infiltrate of foamy macrophages (Virchow cells) in the endoneurial area, and to a lesser extent in the perineurium, containing a faintly eosinophilic amorphous material which was monorefringent on examination with polarised light. Fite-Faraco modification of the Ziehl-Neelsen stain revealed occasional acid-fast bacilli in both endoneurial and perineurial areas, all totally non-solid staining (that is fragmented or granular), but also confirmed the presence of the amorphous material noted above, and showed that it was slightly acid and alcohol fast (fig 1). The only drugs the patient was known to have received for leprosy were dapsone and rifampicin, and at this stage it was thought the unusual appearances might have been due to the destruction in situ of large numbers of acid-fast bacilli, perhaps in globus form, by a penetrating drug (rifampicin). The totally amorphous nature of the material, however, and the fact that it stained rather poorly with a modified Ziehl-Neelsen, led to the additional

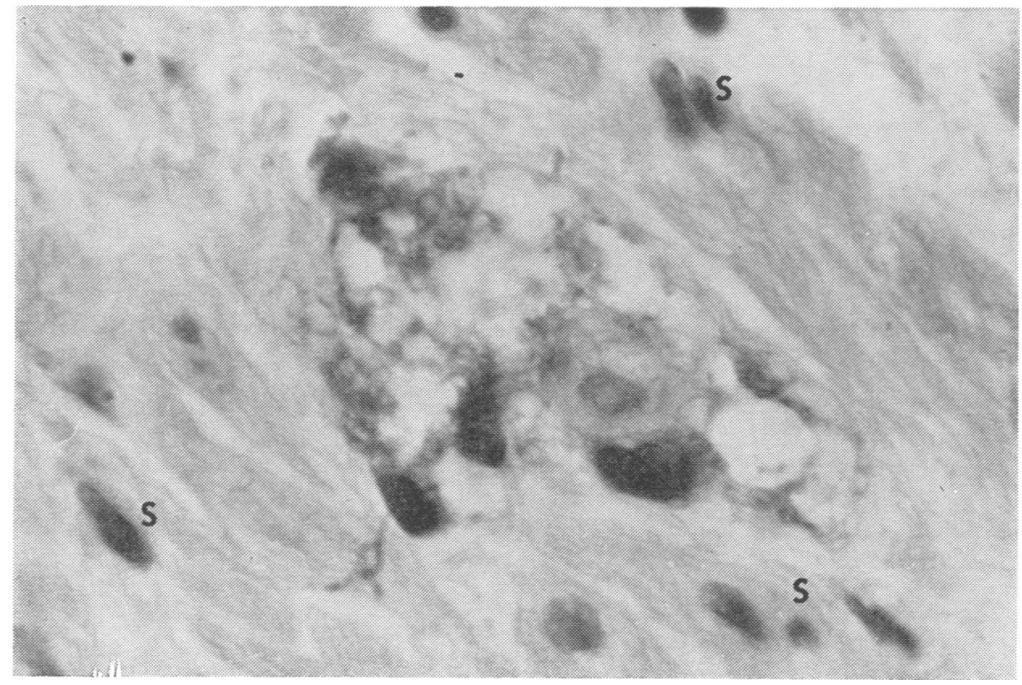

Fig 1 Superficial peroneal nerve; transverse section. In the substance of the endoneurial area, there is a mass of foam (Virchow) cells, the vacuoles of which contain an accumulation of amorphous, faintly acid and alcohol-fast material. $S=$ nuclei of Schwann cells. Fite-Faraco modification of Ziehl-Neelsen stain $(\times 600)$ 
staining procedures which are listed in the table. PAS was weakly positive. Long Ziehl-Neelsen ${ }^{9}$ was strongly positive (fig 2) but did not distinguish the material under examination from bacillary remnants. Sudan Black B, however, using an overnight staining technique, and comparing with a known positive section from a patient with lepromatous leprosy, showed the amorphous material to be strongly sudanophilic (fig 3), whereas leprosy bacilli were negative. Masson-Fontana (ammoniacal silver nitrate) gave similar results; Giemsa stain gave a blue-green colour. Schmorl's ferricyanide technique, usually positive for lipofuscin, was negative, and this result will be discussed below. Finally, congo red and crystal violet for amyloid were both negative, as also was Lendrum's MSB for fibrin. It was considered that these findings were strongly in favour of the material being lipofuscin, or a closely related substance. The unlikelihood of lipofuscin being present in such quantities, and in the foamy cytoplasm of macrophages in nerve, now led to a more challenging enquiry about previous treatment, and the patient was in fact able to produce records showing that he had received treatment with both dapsone and clofazimine in 1976-77, for a period of at least six months-apparently without being informed of the diagnosis of leprosy. Dapsone had been given in a dose of $50-100 \mathrm{mg}$ daily and clofazimine in a dose of $100 \mathrm{mg}$ three times weekly. At

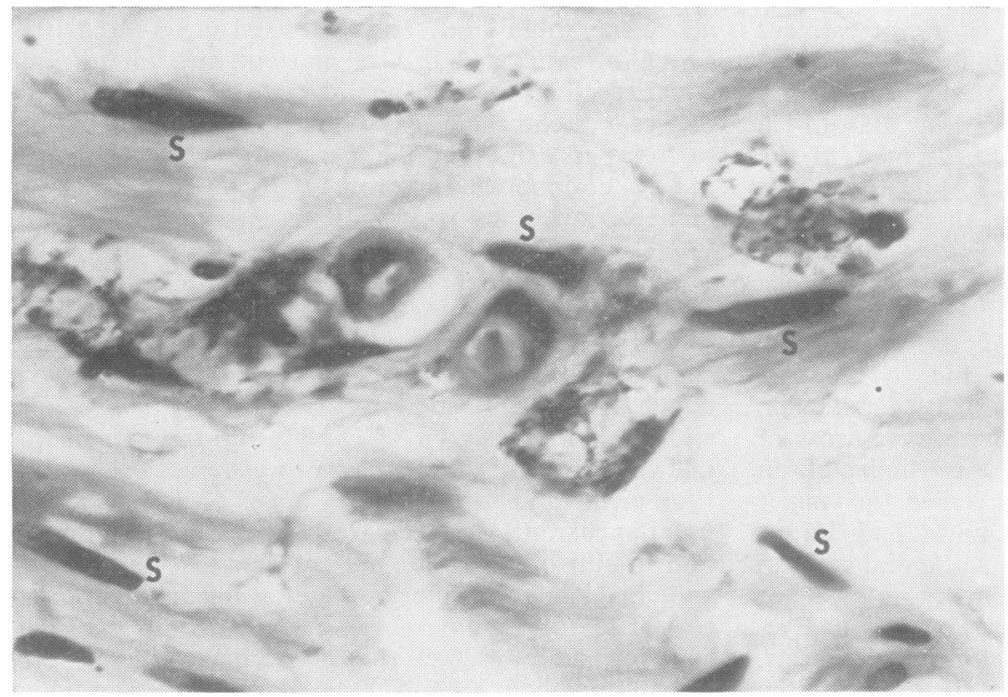

Fig 2 Superficial peroneal nerve; longitudinal section. After long staining with ZiehlNeelsen (3 hours' carbol fuchsin at $60^{\circ} \mathrm{C}$ ), amorphous masses in the cytoplasm of foam cells, similar to those in fig 1 , are stained deeply red. $S=$ nuclei of Schwann cells $(\times 600)$.

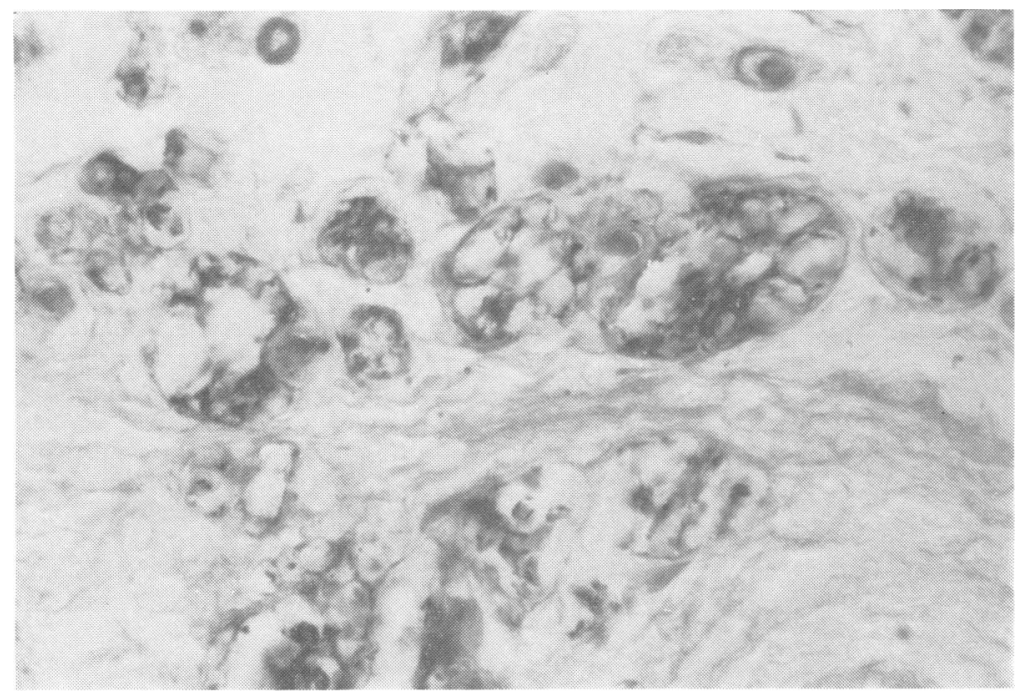

Fig 3 Superficial peroneal nerve; longitudinal section. After staining with Sudan Black $B$ overnight, amorphous masses in the cytoplasm of foam cells stain intensely black. Following fixation in formalin, this biopsy had been processed routinely for wax embedding (X600). 
Table Staining procedures used for the microscopic identification of the amorphous, acid-fast material described in the text

\begin{tabular}{ll}
\hline Technique & Result \\
\hline Haematoxylin and eosin & Weakly eosinophilic \\
Fite-Faraco modification of Ziehl-Neelsen & $\begin{array}{l}\text { Weakly positive } \\
\text { Weakly positive }\end{array}$ \\
Periodic acid-Schiff (PAS) & Strongly positive \\
Long Ziehl-Neelsen & Strongly positive \\
Sudan Black B & Blue-green \\
Giemsa & Negative \\
Schmorl's Ferricyanide & Negative for amyloid \\
Congo Red & Negative for amyloid \\
Crystal Violet & Negative for fibrin \\
Lendrum's MSB & Monorefringent \\
Polarised light & \\
(unstained paraffin sections) & Gold autofluorescence \\
Ultraviolet light & \\
(unstained paraffin sections) & \\
\hline
\end{tabular}

the end of this period he consulted another physician who stopped anti-leprosy treatment, changing to indomethacin on account of persistent "rheumatic" pains in the limbs; he had no further anti-leprosy treatment until mid-1979.

\section{Discussion}

The histochemistry of lipids in leprosy has been extensively investigated" with particular attention to the findings in the macrophages of patients with lepromatous leprosy treated with clofazimine. These studies confirmed that skin biopsies from clofazimine-treated patients retained a reddishbrown pigmentation throughout the dermis, even after the removal of lipids and lipophilic clofazimine from the tissue by fat solvents. They, and other investigators, have demonstrated that this residual coloration is distinct from that which may be caused by the actual deposition of clofazimine, which is a red dye, in tissues, some of which may be in crystal form. Lipofuscins are breakdown products of lipids, formed by their progressive oxidation, the degree of oxidation being arbitrarily divided into "early" and "late". Ceroid, first described by Lillie et $a l^{10}$ is thought to be a lipid in the early stages of oxidation, it retains some of its original lipid-staining properties even after paraffin wax embedding; it is thus strongly sudanophilic (fig 3). The negative Schmorl's reaction (depending on an ability to reduce potassium ferricyanide) is in keeping with the finding in Lillie's original publication, quoted above, and may relate to the concept of ceroid as being at an early stage of lipid oxidation. The strongly positive reaction to long Ziehl-Neelsen staining (fig 3) is typical for ceroid. Although we were unable to confirm the rather striking electronmicroscopic appearances of ceroid ${ }^{11} 12$ due to the lack of suitably fixed material, it is nevertheless considered that the above findings are in keeping with the presence of this substance within nerve.

This pigment is seen quite frequently by histopathologists who monitor skin biopsies of patients treated with this drug, and has not so far been associated with any irritant or other adverse effect on the tissues. On the contrary, clofazimine, despite the disadvantage of skin pigmentation in light-skinned subjects, and the hazards of crystal accumulation if used in high dosage for too long, is currently rated by leprologists as outstandingly valuable in the treament both of the bacillary infection and the suppression of immune complex reactions. The recognition of ceroid-like pigment in biopsies by the procedures described here may be of value in pin-pointing previous drug treatment with clofazimine, and in the accurate assessment of material which is acid and alcohol-fast, but not solely of bacillary origin. A further point of interest is that the findings strongly suggest that this anti-leprosy drug is either penetrating, or being carried into, the endoneurial and perineurial areas of nerve, which often harbour large numbers of Mycobacterium leprae.

The authors are grateful to Dr NR Rowell of the General Infirmary, Leeds, for permission to publish, and to the British Leprosy Relief Association (LEPRA) for financial support.

\section{References}

1 Barry VC, Belton JG, Conalty ML, Denneny JM, Edward DW, O'Sullivan JF, Twomey D, Winder F. A new series of phenazine (rimino-compounds) with high anti-tuberculosis activity. Nature 1957; 179: $1013-15$.

2 Hastings RC, Jacobsen RR, Trautman JR. Longterm clinical toxicity studies with clofazimine (B663) in leprosy. Int $J$ Lep 1976; 44:287-93.

3 Yawalker SJ, Vischer W. Lamprene (clofazimine) in leprosy. Basic information: Pharma Division, Ciba-Geigy Ltd, Basle, Switzerland. Lep Rev 1979; 50:135-44.

4 WHO. WHO Expert Committee on leprosy. Fifth report. Technical Report Series 607. 1977.

5 Jopling WH. Complications of treatment with clofazimine (Lamprene; B663). Editorial. Lep Rev 1976; 47:1-3.

6 Sakurai I, Skinsnes OK. Lipids in leprosy. 2: Histochemistry of lipids in human leprosy. Int J Lep 1970; 38:389-403.

7 Edwards OM, Courtenay-Evans RJ, Galley JM, Hunter J. Changes in cortisol metabolism following rifampicin therapy. Lancet 1974; 2:549-51.

8 Buffington GA, Dominguez JH, Piering WF, Kauffman HM, Lemann J, Jr. Interaction of 
rifampicin and glucocorticoids. Adverse effect on renal allograft function. JAMA 1976; 236:1958-60.

9 Pearse AGE. Histochemistry Theoretical and Applied 2nd ed. Churchill: London, 1960.

10 Lillie RD, Daft FS, Sebrell WHJ. Cirrhosis of liver in rats on deficient diet and effect of alcohol. Publ Health Rep Wash 1941; 56:1255-8.
11 Gyorkey F, Shimamura T, O'Neal RM. The fine structure of ceroid in human atheroma. $J$ Histochem Cytochem 1967; 15:732-6.

12 Amazon K, Rywlin AM. Ceroid granulomas of the gall bladder. Am J Clin Pathol 1980; 73: 123-7. 